\title{
SCIENTOMETRIC PORTRAIT OF T. S. WEST
}

\author{
V. L. KALYANE," S. S. MLNNOLLI** \\ - Tuming Point Centre 1:3, C5-28, Sector 5 C. B. D. Belapur, \\ Konkan Bhavan, New Bombay - 400614 (India) \\ ** Indian Institute of Geomagnetism Dr. Nanabhai Moos Marg, \\ Colaba, Bombay - 400005 (India)
}

(Received January 16, 1995)

\begin{abstract}
T. S. West, the internationally well known analytical chemist has been widely recognised as a very successful scientist. His research productivity and collaboration pattem were analysed by years, papers, authorships, and authorwise productivity. The channels of communications used and distribution of articles among channels were found out. He has 410 papers to his credit. The period $1969-70$ when he was $42-43$ years age was most productive with 41 papers in 1969 and seven single authorship papers in 1970. Quifnquennial collaboration coefficients ranged between 0.57 to 1.00 , clearly indicating high collaboration team spirit in his research group. His productivity coefficient was 0.45 indicating rapid publication activity during early period of research career. His most prominent collaborators in number of papers were: R. M. Dagnall (92), G. F. Kirkbright (77), R. Belcher (56), K. C. Thompson (19), J. D. Viorris. (13), and J. F. Alder (11). Top ranking journals, with papers, to which he had contributed were: Anal. Chim. Acta (106), Talanta (84), The Analyst (49), Anal. Chem. (23), and J. Chem. Soc. (20). Publication density was 8.54 , publication concentration was 6.25 , and average Bradford multiplier was 3.9. High frequency keywords in the titles of the articles were: Atomic fluorescence spectroscopy (51), Atomic absorption spectroscopy (43), and Atomic absorption spectrometry (31). The resuits indicate his temporal publication productivity and the nature of the research activities were such that he is eminently qualified to be taken as a 'role model' for the younger generation to emulate.
\end{abstract}

\section{Introduction}

Thomas Summers West was born at Peterhead, Scotland on 18th November 1927. He had been interested in Analytical Chemistry during school days, and his future scientific path was confirmed while he was living at the YMCA in Aberdeen, where he met Ronald Belcher, Lecturer in Inorganic and Analytical Chemistry at the University. When Belcher moved with Prof. H. W. Melville to the University of Birmingham in 1948, West followed in 1949. There he actively participated in developing the nucleus of Belcher's Birmingham School of Analytical Chemistry. 
At Birmingham Belcher built up an international centre for microanalysis. West's early contribution to that was his work on a quartz fibre torsion ultramicrobalance and on a system of ultramicro organic elemental and functional group methodology. With the award of $\mathrm{Ph}$. D. in 1952 he started up his own group, persuing an independent line of research into Analytical Chelate Chemistry. Also, he did exploratory work on solvent extraction and polarography. He was awarded a Senior DSIR Fellowship (1952-1955), and then became a Lecturer in Chemistry (1955-1963). At Imperial College London he became Reader in Analytical Chemistry during 1963-65. His D. Sc. was conferred in 1962. The research highlight included the specific chromogenic ternary complex reaction between fluoride, either of the first two members of the lanthanides and 3-dicarboxymethylaminomethyl-1, 2-dihydroxyanthraquinone, and the interpretation of the mode of action of this only known positive colour reaction of the fluoride ion. It has subsequently replaced all other spectrophotometric reactions for fluoride. Another important discovery was the unique conditionally specific reaction between calcium and a new specially synthesized trishydroxytriazo clathrate cage dyestuff molecule.

Together with three young post-doctorates from Birmingham-Roy Dagnall, Bernard Fleet, and Gordon Kirkbright - he quickly set up a thriving team, and in 1965 was promoted to the position of Professor of Analytical Chemistry. His work was centred in Analytical Atomic and Molecular Absorption and Fluorescence Spectroscopy. He extended Atomic Absorption Spectrophotometry to pioneering studies in Atomic Spectroscopy by Carbon Rod Electrothermal Automization, Microwave Excited High Intensity Atomic Line Sources, Low Emmissitivity Separated Flames, Chemiluminescence in Diffusion Flames, Atomic Fluorescence Spectroscopy, and Atom Trapping Atomic Absorption Spectroscopy.

In 1975 West returned to Scotland as Director of the Macaulay Institute for Soil Research at Aberdeen. He made contributions to Piezo-electric crystal sensor systems for minute traces of airborne pollutants and to the study of interfacial optical absorption and emission phenomena at platinum electrode surfaces under conditions of diffusion controlled electrolysis in aqueous solutions. Work on biosignificant and toxic trace elements in the soil-plant ecosystem and acidification phenomena led to his participation in the U. K. Royal Society's Surface Water Acidification Programme team together with the Royal Academies of Science in Norway and Sweden. He accepted Honorary Research Professorship of University of Aberdeen in 1983.

West encouraged open thinking and experimentation in new fields due to which many of his students became leaders in many fields of analytical science and in 
domains well away from Chemistry. He played a prominent role in negotiation which led to the amalgamation in the U.K. of the Chemical Society, and Society of Analytical Chemistry. A special issue of the esteemed journal Talanta (Vol. 39, No. 11) was published in 1992 to honour T. S. West, as a teacher, scholar, administrator, researcher, innovator, friend, and colleague.

He established international reputation and received wide spread international recognition and awards. Within the Royal Society of Chemistry West received the Meldola Medal (1956), Perkin-Elmer Medal for Instrumentation and Analytical Chemistry (1977), and Redwood Lectureship (1974). Overseas awards include the Interan Medal (1977) of the Czechoslovak Chemical Society, Johannes Marcus Medal for Spectroscopy (1977) of the Spectroscopic Society of Bohemia, and Honorary Membership (1981) of the Japan Society of Analytical Chemistry. He became a Fellow (1979) of the Royal Society of Edinburgh and in 1989 a Fellow of the Royal Society of London. In the Queen's New Year's Honours List for 1988 he was made a Commander of the Order of the British Empire (CBE) for his contributions to chemistry and international science.

He was associated with International Union of Pure and Applied Chemistry (IUPAC) since 1963, rising to the position of President of the Analytical Chemistry Division (1977-81). After a period as Assistant Secretary General (1981-83), he was elected Secretary General of the Union and served two four-year periods (1983-91). In 1990-92 West became Chairman of the Standing Finance Committee of ICSU and a member of the Executive Board. He has also contributed significantly to other aspects of national and international science.

The development of Analytical Chemistry in the USA is logically described in four time periods, namely (a) the nineteenth century, (b) 1900-1939, (c) the decade of the 1940s, and (d) from 1950 to the present day. ${ }^{1}$ Analytical chemistry has long been, and continues to be, an important component of the undergraduate Bachelor of Science Chemistry degree in most colleges and universities in the USA. ${ }^{2}$

Objectives of the present study were to know authorship pattern-paperwise, yearwise, authorwise, and author productivity; and to find out core channels of communications used, distribution of articles among channels, and Bradford multiplier etc. 


\section{Methodology}

Scientometric analysis is usually based on the utilization of several independent data like publications, citations, experts opinions, content-analysis, etc., which together permit to enlighten various sides of the development of a definite science field. In this paper the publication productivity is used for the analysis.

The choice of the unit of analysis and of the initial set has a strong influence on the measures and results of any bibliometric study. Generally speaking authors are used when the study focuses on the influence of individuals, articles are used to study the influence of a particular idea as embodied in the article, key terms are used to follow an idea over time as it crosses disciplines and journals when the study focuses on the institutional embodiment of a discipline. ${ }^{3}$

Bibliographic details of all publications of $T$. S. West were documented on cards and sorting was done as per requirement to extract various data. Normal count procedure ${ }^{4}$ was followed. Full credit was given to each author regardless of whether he happens to be the first or the last author. It is widely recognised that scientists all over the world look at their own papers exclusively in that way. Similarly titles of the articles were analysed and one score was allotted for each journal, and keyword.

The degree of : collaboration 5 was defined as the ratio of the number of collaborative research papers to the total number of research papers published in a discipline during a certain period of time. Vinkler ${ }^{6}$ defined publication density as the ratio of the total number of papers published to the total number of journals in which the papers were published, and publication concentration as the ratio in percentage of the journals containing half of the papers published to the total number of journals in which those papers were published during the period under study.

Bradford's studies focused on "Journal productivity", in the sense of establishing the small core of journals that contributed the most articles to a subject literature and the scatter of articles on the subject over the remainder. ${ }^{7}$ The Bradford bibliograph has a concavity near the origin for the core or nuclear zone and a linearity afterwords till the downward deviation called "Groos droop". 8

Sen and $\operatorname{Gan}^{9}$ have given the formula for productivity coefficient (P.C.) as the ratio of 50 percentile age to the productivity age. 


\section{Results and discussion}

During 1951-92 T. S. West has published 410 communications as shown in Table 1(a). Single authored communications were $52(12.68 \%)$. Two authored communications were 100 (24.39\%). Three authored publications were 212 (51.70\%). Four authored papers to his credit were $40(9.76 \%)$. He has published five $(1.22 \%)$ communications having five authors each and only one (0.25\%) paper having eight authors. Highest number of communications published in one year were $41(10.00 \%)$ in 1969 at the age of 42 as shown in Table 1(b). Highest number of single authorship papers published in one year were seven during 1970 at the age of 43 . His most productive period was 1968 to 1973 during which he had published 190 (43.36\%) 6 papers at the age of 41 to 46 . Overall the range in Quinquennial Collaboration Coefficients was 0.57 to 1.00 clearly indicating his high collaboration team spirit. Quinquennial publication productivity and related parameters are depicted in Fig. 1.

Table 1(a)

Authorshipwise distribution of papers of T.S. West

\begin{tabular}{rrrrrrrr}
\hline Authors & $\begin{array}{c}\text { No. of } \\
\text { papers }\end{array}$ & $\begin{array}{c}\text { Cumu- } \\
\text { lative } \\
\text { papers }\end{array}$ & $\begin{array}{c}\% \text { of } \\
\text { papers }\end{array}$ & $\begin{array}{c}\text { Cumu. } \\
\text { lative } \\
\%\end{array}$ & $\begin{array}{c}\text { Author- } \\
\text { ships }\end{array}$ & $\begin{array}{c}\% \text { of } \\
\text { author- } \\
\text { ships }\end{array}$ & $\begin{array}{c}\text { Cumulative } \\
\text { author- } \\
\text { ships }\end{array}$ \\
\hline & 52 & 52 & 12.68 & 12.68 & 52 & 4.81 & 52 \\
II & 100 & 152 & 24.39 & 37.07 & 200 & 18.50 & 252 \\
III & 212 & 364 & 51.70 & 88.77 & 636 & 58.84 & 888 \\
IV & 40 & 404 & 9.76 & 98.53 & 160 & 14.80 & 1048 \\
V & 5 & 409 & 1.22 & 99.75 & 25 & 2.31 & 1073 \\
VIII & 1 & 410 & 0.25 & 100.00 & 8 & 0.74 & 1081 \\
\hline
\end{tabular}

$I$ = Single author papers, $I I=$ Two author papers, III = Three author papers, $I V=$ Four author papers $\mathrm{V}=$ Five author papers, VIII = Eight author papers. 
Table 1(b)

Yearwise authorship pattern of T. S. West

\begin{tabular}{|c|c|c|c|c|c|c|c|c|c|c|c|c|}
\hline Year & & II & III & IV & V & VIII & Total & $\begin{array}{c}\text { Cumula- } \\
\text { tive } \\
\text { Total }\end{array}$ & $\%$ & $\begin{array}{c}\text { Cumula- } \\
\text { tive } \%\end{array}$ & $\mathrm{CC}$ & $\begin{array}{l}\text { Age of } \\
\text { T.S. West }\end{array}$ \\
\hline 1951 & - & 8 & & & & & 8 & 8 & 1.95 & 1.95 & 1.00 & 24 \\
\hline 1952 & - & 3 & & & & & 3 & 11 & 0.73 & 2.68 & 1.00 & 25 \\
\hline 1953 & - & & & & & & & 12 & 0.24 & 2.92 & 1.00 & 26 \\
\hline 1954 & 2 & & 3 & & & & 5 & 17 & 1.22 & 4.14 & 0.60 & 27 \\
\hline 1955 & & & 3 & & & & 4 & 21 & 0.98 & 5.12 & 0.75 & 28 \\
\hline 1956 & & & 2 & & & & 3 & 24 & 0.73 & 5.85 & 0.67 & 29 \\
\hline 1957 & & - & 4 & & & & 4 & 28 & 0.98 & 6.83 & 1.00 & 30 \\
\hline 1958 & & 1 & 10 & 2 & & & 14 & 42 & 3.42 & 10.25 & 0.93 & 31 \\
\hline 1959 & & 2 & 7 & - & & & 10 & 52 & 2.44 & 12.69 & 0.90 & 32 \\
\hline 1960 & & 4 & 3 & & & & 8 & 60 & 1.95 & 14.64 & 0.88 & 33 \\
\hline 1961 & 2 & 4 & 3 & & & & 11 & 71 & 2.68 & 17.32 & 0.82 & 34 \\
\hline 1962 & & 7 & 2 & & & & 10 & 81 & 2.44 & 19.76 & 0.90 & 35 \\
\hline 1963 & - & 3 & 3 & & & & 6 & 87 & 1.46 & 21.22 & 1.00 & 36 \\
\hline 1964 & - & 3 & 2 & & & & 5 & 92 & 1.22 & 22.44 & 1.00 & 37 \\
\hline 1965 & 2 & 2 & 13 & & & & 17 & 109 & 4.15 & 26.59 & 0.88 & 38 \\
\hline 1966 & 3 & & 16 & & & & 20 & 129 & 4.88 & 31.47 & 0.86 & 39 \\
\hline 1967 & 4 & 1 & 11 & & & & 17 & 146 & 4.15 & 35.62 & 0.76 & 40 \\
\hline 1968 & - & 6 & 19 & 5 & . & & 30 & 176 & 7.32 & 42.94 & 1.00 & 41 \\
\hline 1969 & 4 & 7 & 23 & 7 & . & & 41 & 217 & 10.00 & 52.94 & 0.90 & 42 \\
\hline 1970 & 7 & 8 & 16 & 5 & - & & 36 & 253 & 8.78 & 61.72 & 0.81 & 43 \\
\hline 1971 & 4 & 5 & 9 & 5 & - & & 23 & 276 & 5.61 & 67.33 & 0.83 & 44 \\
\hline 1972 & & 8 & 20 & 3 & 2 & & 33 & 309 & 8.05 & 75.38 & 1.00 & 45 \\
\hline 1973 & $\dot{5}$ & 9 & 10 & 3 & . & & 27 & 336 & 6.59 & 81.97 & 0.81 & 46 \\
\hline 1974 & & 5 & 7 & & $\cdot$ & & 13 & 349 & 3.17 & 85.14 & 0.92 & 47 \\
\hline 1975 & - & & & & - & & 3 & 352 & 0.73 & 85.87 & 1.00 & 48 \\
\hline 1976 & 3 & & & 2 & & & 7 & 359 & 1.71 & 87.58 & 0.57 & 49 \\
\hline
\end{tabular}


Table 1(b) (Cont.)

\begin{tabular}{|c|c|c|c|c|c|c|c|c|c|c|c|c|}
\hline Year & & II & III & IV & v & VIII & Total & $\begin{array}{c}\text { Cumula- } \\
\text { tive } \\
\text { Total }\end{array}$ & $\%$ & $\begin{array}{l}\text { Cumula- } \\
\text { tive \% }\end{array}$ & $\mathrm{CC}$ & $\begin{array}{c}\text { Age of } \\
\text { T.S. West }\end{array}$ \\
\hline 1977 & - & & 4 & & & & 6 & 365 & 1.46 & 89.04 & 1.00 & 50 \\
\hline 1978 & 2 & & 2 & & & & 6 & 371 & 1.46 & 90.50 & 0.67 & 51 \\
\hline 1979 & 2 & 3 & 2 & & & & 9 & 380 & 2.20 & 92.70 & 0.78 & 52 \\
\hline 1980 & - & 2 & 4 & & & & 6 & 386 & 1.46 & 94.16 & 1.00 & 53 \\
\hline 1981 & 2 & & 2 & & & & 6 & 392 & 1.46 & 95.62 & 0.67 & 54 \\
\hline 1982 & & & 6 & & & & 8 & 400 & 1.95 & 97.57 & 0.88 & 55 \\
\hline 1983 & & & 2 & & & & 2 & 402 & 0.49 & 98.06 & 1.00 & 56 \\
\hline 1984 & & & - & & & - & - & 402 & - & & - & 57 \\
\hline 1985 & & & & & & - & 1 & 403 & 0.24 & 98.30 & 1.00 & 58 \\
\hline 1986 & & & & & & 1 & 5 & 408 & 1.22 & 99.52 & 0.80 & 59 \\
\hline 1987 & & & & - & & - & - & 408 & - & - & - & 60 \\
\hline 1988 & & & & - & & - & & 409 & 0.24 & 99.76 & 1.00 & 61 \\
\hline 1989 & & - & & - & & - & & 409 & - & - & - & 62 \\
\hline 1990 & & - & & - & & - & & 409 & - & - & - & 63 \\
\hline 1991 & & - & & - & & - & & 409 & - & - & - & 64 \\
\hline 1992 & & - & & - & - & - & & 410 & 0.24 & 100.00 & 0.00 & 65 \\
\hline Total & 52 & 100 & 212 & 40 & 5 & & 410 & & & & & \\
\hline Cumu- & & & & & & & & & & & & \\
\hline lative & 52 & 152 & 364 & 404 & 409 & 410 & & & & & & \\
\hline & 12.68 & 24.39 & 51.70 & 9.76 & 1.22 & 0.25 & & & & & & \\
\hline $\begin{array}{l}\text { Cumu- } \\
\text { lative }\end{array}$ & 12.68 & 37.07 & 88.77 & 98.53 & 99.75 & 100.00 & & & & & & \\
\hline
\end{tabular}

$\mathrm{I}=$ Single author papers, II $=$ Two author papers, III $=$ Three author papers, IV = Four author papers, $\mathrm{V}=$ Five author papers, VIII $=$ Eight author papers, $\mathrm{CC}=$ Collaboration Coefficient. 


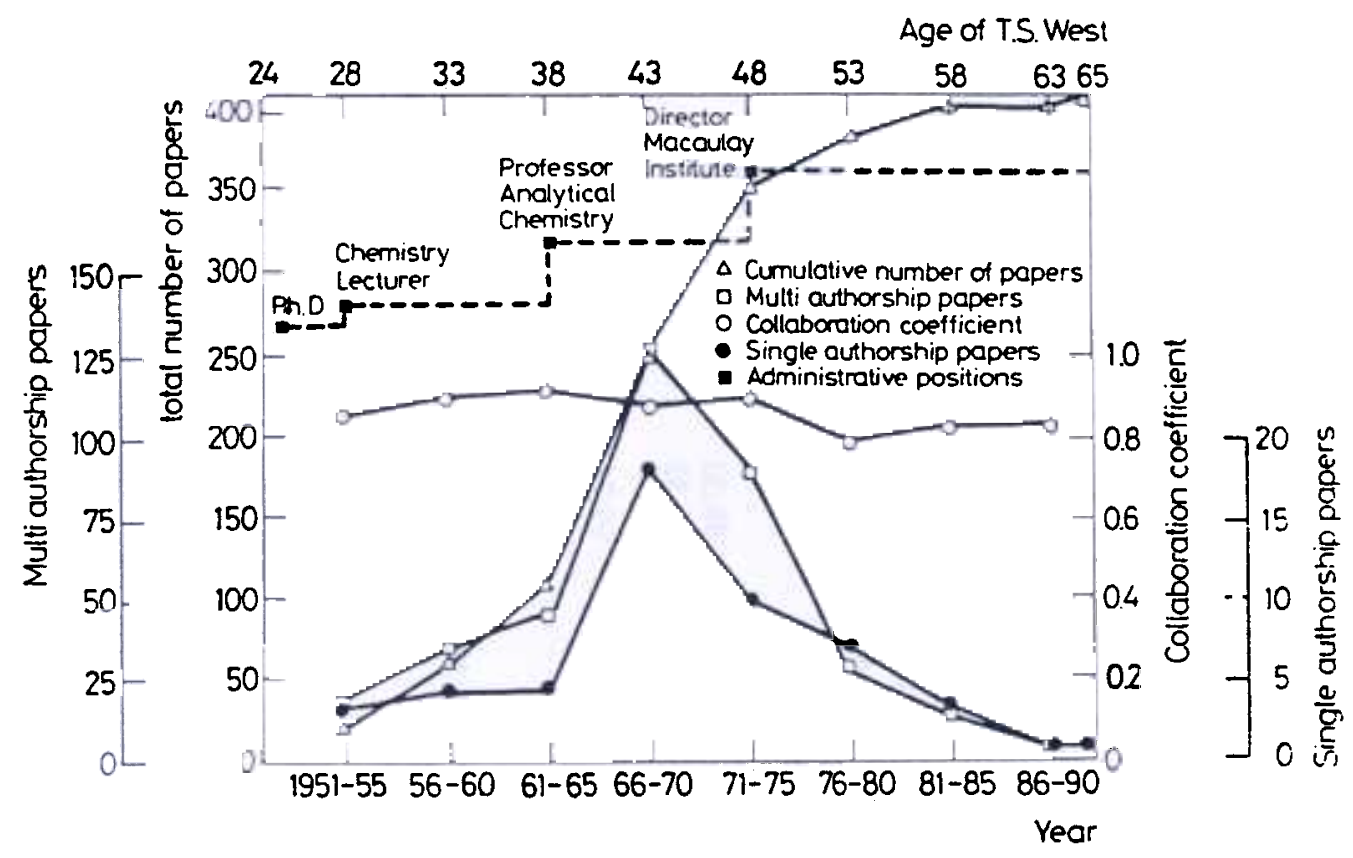

Fig. 1. Yearwise contributions of T. S. West

The "success breeds success" phenomenon has its limits. ${ }^{10}$ Saturation takes place and instead of accelerating of a production rate prolific authors are satisfied with their position and produce less than could be expected from Lotka's law.

T. S. West had productivity coefficient of 0.45 . P.C. values smaller than 0.5 indicate rapid publication in an early period of career. It is said that mathematicians publish more in early life and biologists work more in the middle of their careers.

Authorwise and authorshipwise communication productivity is shown in Table 2(a) and Fig. 2. He has collaborated with 167 other researchers. Total number of authorships were 1081. Prominent collaborators with their authorships were R. M. Dagnall (92), G. F. Kirkbright (77), R. Belcher (56), K. C. Thompson (19), and J. D. Norris (13). The collaborators having ten authorships each were A. J. Aggett, K. M. Aldous, B. L. Sharp, and A. M. Ure. 
Table 2(a)

Authorship pattern of T. S. West and his collaborators

\begin{tabular}{|c|c|c|c|c|c|c|c|c|c|c|c|}
\hline \multirow{2}{*}{$\begin{array}{l}\text { Code } \\
\text { No. }\end{array}$} & \multirow[t]{2}{*}{ Authors } & \multicolumn{6}{|c|}{ Number of Authorships } & \multirow[t]{2}{*}{ Total } & \multicolumn{2}{|c|}{ Year } & \multirow[t]{2}{*}{ Total } \\
\hline & & I & II & III & IV & V & VIII & & FPY & LPY & \\
\hline 1. & West, T.S. & 52 & 100 & 212 & 40 & 5 & & 410 & 1951 & -92 & 42 \\
\hline 2. & Dagnall, R.M. & - & 7 & 60 & 23 & 2 & & 92 & 1961 & .75 & 15 \\
\hline 3. & Kirkbright, G.F. & - & 3 & 60 & 12 & 2 & & 77 & 1965 & -76 & 12 \\
\hline 4. & Belcher, R. & & 14 & 41 & & & & 56 & 1951 & -82 & 32 \\
\hline 5. & Thompson, K.C. & & & 15 & 4 & & & 19 & 1966 & -86 & 21 \\
\hline 6. & Norris, J.D. & & 10 & - & 2 & & & 13 & 1971 & - 79 & 9 \\
\hline 7. & Alder, J.F. & & 4 & 4 & 3 & & & 11 & 1970 & -72 & 3 \\
\hline 8. & Aldous, K.M. & & & 3 & 6 & & & 10 & 1968 & -73 & 6 \\
\hline 9. & Sharp, B.L. & & & 4 & 5 & & & 10 & 1971 & -86 & 16 \\
\hline 10. & Ure, A.M. & & - & 8 & & & & 10 & 1979 & -86 & 8 \\
\hline 11. & Close, R.A. & & 3 & 4 & & & & 7 & 1957 & -60 & 4 \\
\hline 12. & Fleet, B. & & - & 7 & - & & & 7 & 1968 & -71 & 4 \\
\hline 13. & Hoyle, W. & & 2 & 5 & - & & & 7 & 1958 & -62 & 5 \\
\hline 14. & Alger, $D$. & & - & & 6 & & & 6 & 1967 & -76 & 10 \\
\hline 15. & Browner, R.F. & & & 4 & 1 & & & 6 & 1969 & -72 & 4 \\
\hline 16. & Cabrera, A.M. & & 2 & 4 & - & & & 6 & 1962 & -65 & 4 \\
\hline 17. & Cresser, M.S. & & 6 & - & - & & & 6 & 1968 & -73 & 6 \\
\hline 18. & Ebdon, L. & & & 5 & & & & 6 & 1969 & -72 & 4 \\
\hline 19. & Gibbons, D. & & & 6 & & & & 6 & 1954 & -55 & 2 \\
\hline 20. & Sargent, M. & & & 3 & 3 & & & 6 & 1968 & -70 & 3 \\
\hline 21. & Silvester, M.D. & & & 3 & 3 & & & 6 & 1971 & -73 & 3 \\
\hline 22. & Smith, A.M. & & & 5 & & & & 6 & 1966 & -69 & 4 \\
\hline 23. & Smith, R. & & & 5 & 1 & & & 6 & 1965 & -67 & 3 \\
\hline 24. & Wood, R. & & & - & 6 & & & 6 & 1969 & -72 & 4 \\
\hline 25. & Woodward, C. & & & 6 & - & & & 6 & 1965 & -66 & 2 \\
\hline 26. & Young, $P$. & & & 6 & - & & & 6 & 1965 & -67 & 3 \\
\hline 27. & Cooke, D.O. & & & 3 & & & & 5 & 1971 & -72 & 2 \\
\hline
\end{tabular}


Table 2(a) (Cont.)

\begin{tabular}{|c|c|c|c|c|c|c|c|c|c|c|c|}
\hline \multirow{2}{*}{$\begin{array}{l}\text { Code } \\
\text { No. }\end{array}$} & \multirow{2}{*}{ Authors } & \multirow[b]{2}{*}{$\mathbf{I}$} & \multirow[b]{2}{*}{ II } & \multicolumn{3}{|c|}{ Number of Authorships } & \multirow[b]{2}{*}{ VIII } & \multirow[t]{2}{*}{ Total } & \multicolumn{2}{|c|}{ Year } & \multirow[t]{2}{*}{ Total } \\
\hline & & & & III & IV & v & & & FPY & LPY & \\
\hline 28. & Everett, G.L. & & 1 & 4 & & & & 5 & 1973 & -74 & 2 \\
\hline 29. & Leonard, M.A. & & & 4 & & & & 5 & 1958 & -60 & 3 \\
\hline 30. & Medal, A.S. & & & 3 & & & & 5 & 1977 & -79 & 3 \\
\hline 31. & Williams, R.W. & & & 4 & & & & 5 & 1973 & -76 & 4 \\
\hline 32. & Wilson, P.J. & & & 5 & & & & 5 & 1972 & -74 & 3 \\
\hline 33. & Clark, D. & & & 3 & & & & 4 & 1972 & -73 & 2 \\
\hline 34. & Edmonds, T.E. & & & 3 & & & & 4 & 1980 & -86 & 7 \\
\hline 35. & Hingle, D.N. & & - & 3 & 1 & & & 4 & 1968 & - 69 & 2 \\
\hline 36. & Khalighie, J. & & - & 4 & - & & & 4 & 1979 & -82 & 4 \\
\hline 37. & Peters, M.K. & & - & 3 & & & & 4 & 1966 & -68 & 3 \\
\hline 38. & Ramakrishna, T.V. & & - & 4 & - & & & 4 & 1963 & -69 & 7 \\
\hline 39. & Samuel, A.J. & & - & 2 & 2 & & & 4 & 1976 & -77 & 2 \\
\hline 40. & Sanderson, I.P. & & 2 & 2 & - & & & 4 & 1961 & -63 & 3 \\
\hline 41. & Saw, C.G. & & - & 3 & & & & 4 & 1969 & -69 & 1 \\
\hline 42. & Semb, A. & & - & 4 & - & & & 4 & 1967 & -68 & 2 \\
\hline 43. & Whitehead, P. & & - & 3 & 1 & & & 4 & 1972 & -73 & 2 \\
\hline 44. & Win, Soe. & & & 4 & - & - & & 4 & 1968 & -69 & 2 \\
\hline 45. & Anderson, R.G. & & & 2 & & - & & 3 & 1970 & -71 & 2 \\
\hline 46. & Baaz, M. & & & & 1 & 1 & & 3 & 1961 & .61 & \\
\hline 47. & Baily, B.W. & & & 2 & & 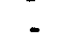 & & 3 & 1965 & -66 & 2 \\
\hline 48. & Bhatty, M.K. & & - & 3 & & - & & 3 & 1957 & -60 & 4 \\
\hline 49. & Budesinsky, B. & & 3 & - & & - & & 3 & 1968 & -69 & 2 \\
\hline 50. & Burriel, F. & & & 3 & & - & & 3 & 1965 & -65 & \\
\hline 51. & El-Ghamry, M.T. & & & 3 & & - & & 3 & 1966 & -68 & 3 \\
\hline 52. & Gutmann, V. & & & 1 & & 1 & & 3 & 1961 & -61 & 1 \\
\hline 53. & Hems, R.V. & & & 3 & & - & & 3 & 1969 & -70 & 2 \\
\hline 54. & Hobbs, R.S. & & - & 2 & & - & & 3 & 1968 & -71 & 4 \\
\hline 55. & Jackson, K.W. & & 2 & & & - & & 3 & 1972 & -73 & 2 \\
\hline
\end{tabular}


Table 2(a) (Cont.)

\begin{tabular}{|c|c|c|c|c|c|c|c|c|c|c|}
\hline \multirow{2}{*}{$\begin{array}{l}\text { Code } \\
\text { No. }\end{array}$} & \multirow[t]{2}{*}{ Authors } & \multirow[b]{2}{*}{ II } & \multicolumn{3}{|c|}{ Number of Authorships } & \multirow[b]{2}{*}{ VIII } & \multirow[t]{2}{*}{ Total } & \multicolumn{2}{|c|}{ Year } & \multirow[t]{2}{*}{ Total } \\
\hline & & & III & IV & v & & & FPY & LPY & \\
\hline 56. & Johnson, H.N. & - & 2 & - & & & 3 & 1971 & -72 & 2 \\
\hline 57. & Lau, C.M. & - & 3 & - & & & 3 & 1982 & -83 & 2 \\
\hline 58. & Liberty, K.V. & - & 3 & - & & & 3 & 1968 & -70 & 3 \\
\hline 59. & Marshall, G.B. & 3 & & - & & & 3 & 1967 & -72 & 6 \\
\hline 60. & MacDonald, A.M.G. & - & 3 & - & & & 3 & 1958 & -65 & 8 \\
\hline 61. & Narayanaswamy, $\mathbf{R}$. & - & 3 & - & & & 3 & 1970 & -72 & 3 \\
\hline 62. & Nomura, $T$. & - & 2 & 1 & & & 3 & 1980 & -85 & 6 \\
\hline 63. & Perez-Bustamante, J.A. & - & 3 & & & & 3 & 1977 & -78 & 2 \\
\hline 64. & Pratt, S.J. & - & 1 & 2 & & & 3 & 1969 & -70 & 2 \\
\hline 65. & Rahim, S. A. & & 2 & - & & & 3 & 1969 & - 74 & 6 \\
\hline 66. & Smith, D.J. & & 2 & & & & 3 & 1969 & -71 & 3 \\
\hline 67. & Tyson, J.F. & 3 & - & & & & 3 & 1974 & -80 & 7 \\
\hline 68. & Williams, X.K. & 3 & & & & & 3 & 1968 & -69 & 2 \\
\hline 69. & Aggett, A.J. & 2 & & & & & 2 & 1971 & -71 & \\
\hline 70. & Berjer, J. & - & 2 & & & & 2 & 1959 & -59 & \\
\hline 71. & Betteridge, D. & 2 & & & & & 2 & 1962 & -62 & \\
\hline 72. & Bhasin, R.L. & - & & & & & 2 & 1958 & -59 & 2 \\
\hline 73. & Chester, J.E. & - & 1 & & & & 2 & 1968 & -70 & 3 \\
\hline 74. & Dowson, W.M. & - & 1 & & & & 2 & 1956 & -58 & 3 \\
\hline 75. & Fraser, S.M. & - & 1 & & & & 2 & 1986 & -86 & \\
\hline 76. & Guogang, Pu. & - & 2 & - & & & 2 & 1980 & -81 & 2 \\
\hline 77. & Harrison, R. & - & & 1 & & & 2 & 1956 & -58 & 3 \\
\hline 78. & Johnson, D.J. & & & - & & & 2 & 1973 & -73 & \\
\hline 79. & King, A.F. & & & - & & & 2 & 1973 & -79 & 7 \\
\hline 80. & Maines, I.S. & & & & & & 2 & 1970 & -71 & 2 \\
\hline 81. & Mansfield, J.M. & & & 1 & & & 2 & 1973 & -74 & 2 \\
\hline 82. & Mayhew, M.D. & & & & & & 2 & 1967 & - 69 & 3 \\
\hline 83. & Mayne, P.J. & & 2 & & & & 2 & 1971 & -72 & 2 \\
\hline
\end{tabular}


Table 2(a) (Cont.)

\begin{tabular}{|c|c|c|c|c|c|c|c|c|c|c|c|}
\hline \multirow{2}{*}{$\begin{array}{l}\text { Code } \\
\text { No. }\end{array}$} & \multirow[t]{2}{*}{ Authors } & \multicolumn{6}{|c|}{ Number of Authorships } & \multirow[t]{2}{*}{ Total } & \multicolumn{2}{|c|}{ Year } & \multirow[t]{2}{*}{ Total } \\
\hline & & 1 & II & III & IV & V & VIII & & FPY & LPY & \\
\hline 84. & Ranson, L. & & - & 1 & & - & - & 2 & 1972 & 73 & 2 \\
\hline 85. & Rao, A.P. & & - & 2 & & - & - & 2 & 1969 & 69 & \\
\hline 86. & Sandell, E.B. & & 2 & - & & - & - & 2 & 1969 & 79 & 11 \\
\hline 87. & Shah, R.A. & & & 1 & & - & - & 2 & 1958 & 58 & \\
\hline 88. & Stephens, R. & & 2 & - & - & : & - & 2 & 1972 & 77 & 6 \\
\hline 89. & Sykes, A.S. & & 2 & - & - & - & - & 2 & 1958 & 61 & 4 \\
\hline 90. & Tan, L.H. & & 2 & - & - & - & - & 2 & 1971 & 71 & 1 \\
\hline 91. & Thompson, J.H. & & - & 2 & - & - & - & 2 & 1958 & 58 & 1 \\
\hline 92. & Ward, A.F. & & - & 2 & - & - & - & 2 & 1972 & 73 & 2 \\
\hline \multirow[t]{2}{*}{$93-168}$. & others $\left(\mathrm{g}^{6}\right)$ having & & 15 & 36 & 14 & 6 & 5 & 76 & & & \\
\hline & & 52 & 200 & 635 & 161 & 25 & 8 & 1081 & & & \\
\hline
\end{tabular}

I = Single author papers, II = two author papers, III, Three author papers, IV = Four author papers, $\mathrm{V}=$ Five author papers, VIII = Eight author papers, FPY - First Paper publication Year and LPY - Last Paper publication Year. 


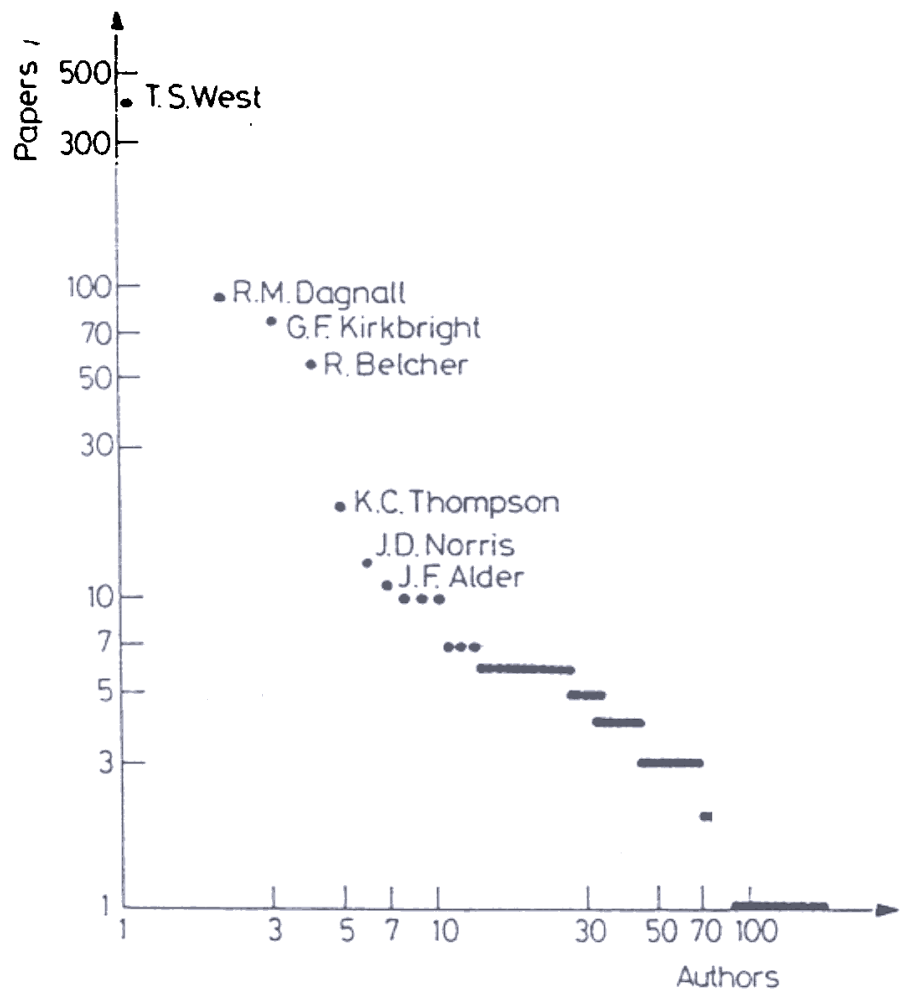

Fig. 2. Publication productivity of T. S. West and collaborators (log-log scale)

Table 2(b)

Author productivity

\begin{tabular}{rrrrr}
\hline Author(s) & Authorship(s) & $\begin{array}{c}\text { Total } \\
\text { authorships }\end{array}$ & Percentage & $\begin{array}{c}\text { Cumulative } \\
\text { percentage }\end{array}$ \\
\hline & & 410 & 37.92 & 37.92 \\
1 & 410 & 92 & 8.51 & 46.43 \\
& 92 & 77 & 7.12 & 53.55 \\
1 & 77 & 56 & 5.18 & 58.73 \\
1 & 56 & 19 & 1.76 & 60.49 \\
3 & 19 & 13 & 1.20 & 62.71 \\
3 & 13 & 11 & 1.02 & 65.49 \\
13 & 11 & 30 & 2.78 & 67.43 \\
6 & 10 & 21 & 1.94 & 74.65 \\
12 & 7 & 78 & 7.22 & 77.43 \\
24 & 6 & 30 & 2.78 & 81.87 \\
24 & 5 & 48 & 4.44 & 92.97 \\
76 & 4 & 72 & 6.66 & 100.00 \\
\hline 168 & 3 & 48 & 4.44 & \\
\hline
\end{tabular}


Table 3(a)

Channels of communications used by T. S. West

\begin{tabular}{|c|c|c|c|c|c|c|c|c|c|}
\hline \multirow{2}{*}{$\begin{array}{l}\text { Sr. } \\
\text { No. }\end{array}$} & \multirow[t]{2}{*}{ Channels of communications } & \multirow{2}{*}{$\begin{array}{l}\text { No. of } \\
\text { papers }\end{array}$} & \multirow{2}{*}{$\begin{array}{l}\text { Cumu- } \\
\text { lative }\end{array}$} & \multirow[t]{2}{*}{$\%$} & \multirow{2}{*}{$\begin{array}{c}\text { Cumu- } \\
\text { lative } \\
\%\end{array}$} & \multicolumn{2}{|c|}{ Year } & \multicolumn{2}{|c|}{ SCI, JCR 1992} \\
\hline & & & & & & FPY & - LPY & $\begin{array}{l}\text { Impact } \\
\text { factor }\end{array}$ & $\begin{array}{c}\text { Immediacy } \\
\text { index }\end{array}$ \\
\hline 1. & Anal. Chim. Acta & 106 & 106 & 25.85 & 25.85 & 1951 & -86 & 2.033 & 0.221 \\
\hline 2. & Talanta & 84 & 190 & 20.48 & 46.33 & 1958 & -83 & 1.236 & 0.126 \\
\hline 3. & The Analyst & 49 & 239 & 11.95 & 58.28 & 1955 & -86 & 1.588 & 0.219 \\
\hline 4. & Anal. Chem. & 23 & 262 & 5.60 & 63.88 & 1954 & -75 & 4.494 & 0.592 \\
\hline 5. & J. Chem. Soc. & 20 & 282 & 4.90 & 68.78 & 1953 & - 72 & & \\
\hline 6. & Books edited \& contributed to & 14 & 296 & 3.45 & 72.23 & 1956 & -92 & & - \\
\hline 7. & Spectroscopy Letters & 10 & 306 & 2.53 & 74.76 & 1968 & -78 & 0.505 & 0.078 \\
\hline 8. & Chemistry \& Industry & 8 & 314 & 1.95 & 76.71 & 1954 & -70 & 0.369 & 0.417 \\
\hline 9. & Proc. of Symp.,Conf.,Congress, meet & 8 & 322 & 1.95 & 78.66 & 1958 & -82 & & \\
\hline 10. & Anal.Quim.Real.Soc.Espan.Fis.Y.Quim. & 1. 7 & 329 & 1.70 & 80.36 & 1963 & -77 & & \\
\hline 11. & Lab Practice & 7 & 336 & 1.70 & 82.06 & 1965 & -76 & & \\
\hline 12. & Proc. Soc. Analyt. Chem. & 7 & 343 & 1.70 & 83.76 & 1972 & $-88^{\prime} 0$ & & - \\
\hline 13. & Pure \& Appl. Chem. & 6 & 349 & 1.46 & 85.22 & 1969 & -79 & 1.739 & 0.161 \\
\hline 14. & Spectrochim. Acta & 5 & 354 & 1.21 & 86.43 & 1970 & -73 & 0.806 & 0.144 \\
\hline 15. & Serials edited & 4 & 358 & 0.97 & 87.40 & 1973 & -76 & - & - \\
\hline 16. & Anal. Lett. & 3 & 361 & 0.73 & 88.13 & 1969 & -70 & 1.000 & 0.062 \\
\hline 17. & Ann. Repts. Chem. Soc. & 3 & 364 & 0.73 & 88.86 & 1954 & -56 & & \\
\hline 18. & Atomic Absorption Newsletter & 3 & 367 & 0.73 & 89.59 & 1967 & -72 & & - \\
\hline 19. & Mikrochim. Acta & 3 & 370 & 0.73 & 90.32 & 1956 & -74 & 0.795 & 0.214 \\
\hline 20. & Patents & 3 & 373 & 0.73 & 91.05 & 1969 & -73 & & \\
\hline 21. & Anal. Proc. & 2 & 375 & 0.48 & 91.53 & 1982 & -82 & & - \\
\hline 22. & Applied Optics & 2 & 377 & 0.48 & 92.01 & 1968 & -68 & 1.064 & 0.210 \\
\hline 23. & Bunseki Kagaka & 2 & 379 & 0.48 & 92.49 & 1981 & -81 & 0.382 & 0.038 \\
\hline 24. & Chemist Analyst & 2 & 381 & 0.48 & 92.97 & 1957 & - 58 & 0.323 & 0.054 \\
\hline 25. & Chem. in Britain & 2 & 383 & 0.48 & 93.45 & 1971 & -72 & 1.485 & 0.896 \\
\hline 26. & J. Electroanal. Chem. & 2 & 385 & 0.48 & 93.93 & 1961 & -69 & 2.202 & 0.200 \\
\hline 27. & Minerals Science \& Engineering & 2 & 387 & 0.48 & 94.41 & 1969 & -70 & & \\
\hline 28. & Monatsh. f. Chemie. & 2 & 389 & 0.48 & 94.89 & 1961 & -61 & 0.471 & 0.132 \\
\hline 29. & Nature & 2 & 391 & 0.48 & 95.37 & 1972 & -74 & 22.139 & 5.224 \\
\hline $30-48$ & others (19) one each & 19 & 410 & 4.63 & 100.00 & 1954 & -86 & & \\
\hline
\end{tabular}

FPY = First Paper publication year, LPY = Last Paper publication Year, SCI = Science Citation Index, and JCR = Journal Citation Report. 
Table 3 (b)

Distribution of articles among channels of communications

\begin{tabular}{rrrrrr}
\hline CH & C & CH.C & ICH & ICH.C & Percentage \\
\hline 1 & 106 & 106 & 1 & 106 & 25.85 \\
1 & 84 & 84 & 2 & 190 & 46.34 \\
1 & 49 & 49 & 3 & 239 & 58.29 \\
1 & 23 & 23 & 4 & 262 & 63.90 \\
1 & 20 & 20 & 5 & 282 & 68.78 \\
1 & 14 & 14 & 6 & 296 & 72.19 \\
1 & 10 & 10 & 7 & 306 & 74.63 \\
2 & 8 & 16 & 9 & 322 & 78.54 \\
3 & 7 & 21 & 12 & 343 & 83.66 \\
1 & 6 & 6 & 13 & 349 & 85.12 \\
1 & 5 & 5 & 14 & 354 & 86.34 \\
1 & 4 & 4 & 15 & 358 & 87.32 \\
5 & 3 & 15 & 20 & 373 & 90.98 \\
9 & 2 & 18 & 29 & 391 & 95.37 \\
19 & 1 & 19 & 48 & 410 & 100.00 \\
\hline
\end{tabular}

$\mathrm{CH}=$ Channels of communications, $\mathrm{C}=$ Communications or number of publications, $\mathrm{CH} . \mathrm{C}=$ Total communications, $\Sigma \mathrm{CH}=$ Cumulative channel and $\Sigma \mathrm{CH} . \mathrm{C}=$ Cumulative total communications.

Productivity of the research group of T. S. West is shown in Table 2(b). The 26 percent top authors (44) have 82 percent authorships to their credit. Remaining 74 percent authors (124) have 18 percent authorships. Hence it follows $80 / 20$ rule. ${ }^{11}$ Sixty percent authorships belong to top ranking, five authors only constituting three percent of the total authors.

Channels of communications used by T. S. West are shown in Table 3(a). He has published through 48 channels of communications. Highest number of papers 106 (25.85\%) were published in Anal. Chim. Acta having impact factor 2.033 and immediacy index 0.221. Talanta has published his 84 (20.49\%) papers. The Analyst has published 49 (11.95\%) papers of T.S. West. The 80.36 percent articles are published in 20.83 percent of channels of communications. Thus following $80 / 20$ rule.

Highest impact factor (22.139) journal Nature having immediacy index 5.224 had published two papers. The Analytical Chemistry journal had published 23 papers (5.61\%) having impact factor 4.494 and immediacy index 0.592 .

Distribution of articles among channels is shown in Table $3(\mathrm{~b})$, which indicates characteristic choice of channels to communicate research results of research group of T. S. West. 


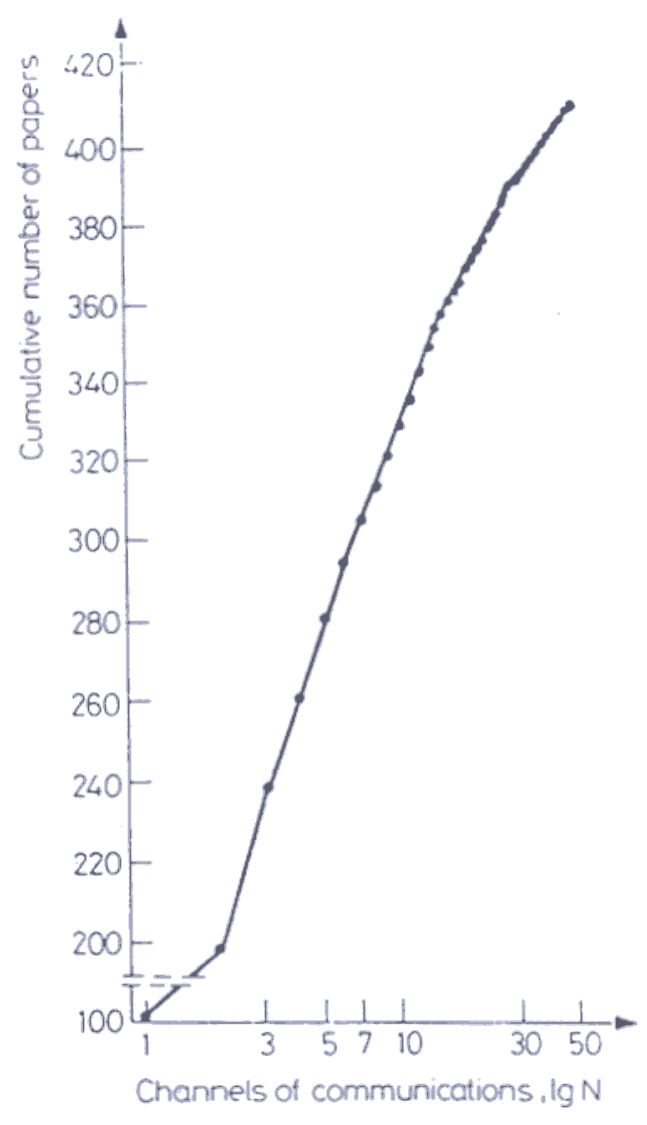

Fig. 3. Bradford-Zipf bibliograph

Bradford-Zipf bibliograph is provided in Fig. 3. The Table 3(c) provides information on Bradford distribution (four zones). Average Bradford multiplier $\bar{b}$ was found to be 3.9. The Bradford's theoretical law has practical consequences for any reader: one should try to find out - using ones own experience, consultation with colleagues or librarians, checking abstracting journals indexes, and references what are the core journals in ones field of interest. One should pay a great deal of attention to those one should probably read or at least scan those cover-to-cover in a library or one should subscribe those. The citations and review columns will lead to other sources outside the core group. Another larger, group of journals, on average, fewer but still a reasonable number of relevant articles (roughly - the second level according to Bradford law) is worth scanning. 
Table 3(c)

Bradford distribution (four zones) of channels of communications used by T. S. West

\begin{tabular}{lccc}
\hline Zone & $\begin{array}{c}\text { No. of } \\
\text { journals }\end{array}$ & $\begin{array}{c}\text { No. of } \\
\text { articles }\end{array}$ & $\begin{array}{c}\text { Bradford } \\
\text { multiplier }\end{array}$ \\
\hline First & 1 & 106 & \\
Second & 2 & 133 & 2.00 \\
Third & 5 & 104 & 2.50 \\
Fourth & 36 & 67 & 7.20 \\
\hline
\end{tabular}

Average Bradford multiplier $(\bar{b})=3.9$.

Keywords from the title of articles were counted and their frequencies are included in Table 4. The results indicate that he had wide ranging interest.

A researcher is good if he or she surpasses the expectations of what he or she might achieve. But this is only possible if the focus is on work and the problem involved, and not on competition or success. The effectiveness of research or of running an institution depends primarily on the researcher himself, on his models, and within the institute itself, on the respect he shows his staff members, which, in turn, enables him to demand that they work to capacity. ${ }^{12}$ Scientists of all disciplines should learn from the humanities.

Table 4

Keyword having two or more frequency in the titles

\begin{tabular}{rlr}
\hline $\begin{array}{r}\text { Sr. } \\
\text { No. }\end{array}$ & \multicolumn{1}{c}{ Keyword } & Frequency \\
\hline 1. & Atomic fluorescence spectroscopy & 51 \\
2. & Atomic absorption spectroscopy & 43 \\
3. & Atomic absorption spectrometry & 31 \\
4. & Spectrophotometry & 23 \\
5. & Spectrofluorimetry & 21 \\
6. & Copper & 19 \\
7. & Carbon filament atom reservoir & 18 \\
8. & Atomic fluorescence spectrometry & 13 \\
9. & Atomic fluorescence spectroscopy & 13 \\
10. & Organic analysis & 12 \\
11. & Silver & 12 \\
12. & Air acetylene flame & 11 \\
13. & Atom reservoir & 11 \\
\hline
\end{tabular}


Table 4 (Cont.)

\begin{tabular}{|c|c|c|c|}
\hline Sr. & Keyword & Frequ & \\
\hline 14. & Calcium & 11 & $\because$ \\
\hline 15. & Lead & 11 & \\
\hline 16. & Nitrogen oxygen acetylene flame & 11 & \\
\hline 17. & Atomic absorption spectrophotometry & 10 & \\
\hline 18. & Carbon & 10 & \\
\hline 19. & Microwave electrodeless discharge tube & 9 & \\
\hline 20. & Niobium & 9 & \\
\hline 21. & Selenium & 9 & \\
\hline 22. & Sulphur & 9 & \\
\hline 23. & Aluminium & 8 & \\
\hline 24. & Electrodeless discharge tube & 8 & \\
\hline 25. & Mercurous nitrate & 8 & \\
\hline 26. & Molybdenum & 8 & \\
\hline 27. & Phosphorus & 8 & \\
\hline 28. & Reductimetric reagent & 8 & \\
\hline 29. & Spectroscopy & 8 & \\
\hline 30. & Ternary complexes & 8 & \\
\hline 31. & Zinc & 8 & \\
\hline 32. & Analytical chemistry & 7 & \\
\hline 33. & Chromium & 7 & \\
\hline 34. & Gas-phase chromatography & 7 & \\
\hline 35. & Iodine & 7 & \\
\hline 36. & Manganese & 7 & \\
\hline 37. & Mercury & 7 & \\
\hline 38. & Antimony & & \\
\hline 39. & Arsenic & & \\
\hline 40. & Cadmium & 6 & \\
\hline 41. & Soil science & 6 & \\
\hline 42. & Atomic spectroscopy & 5 & \\
\hline 43. & Aqueous solution & 5 & \\
\hline 44. & Beryllium & 5 & \\
\hline 45. & Fluorescence & 5 & \\
\hline 46. & Magnesium & 5 & \\
\hline 47. & Nickel & 5 & \\
\hline 48. & Nitrogen & 5 & \\
\hline 49. & Nitrous oxide acetylene flames & 5 & \\
\hline 50. & Polarography & 5 & \\
\hline 51. & Steel & 5 & \\
\hline 52. & Thermal emission spectroscopy & 5 & \\
\hline 53. & Vanadium & 5 & \\
\hline 54. & Atomic emission spectrometry & 4 & \\
\hline 55. & Bismuth & 4 & \\
\hline 56. & Cations & 4 & \\
\hline 57. & Chelometry & 4 & \\
\hline
\end{tabular}


Table 4 (Cont.)

\begin{tabular}{|c|c|c|}
\hline $\begin{array}{l}\text { Sr. } \\
\text { No. }\end{array}$ & Keyword & Frequency \\
\hline 58. & Cobalt & 4 \\
\hline 59. & Fluorescence spectroscopy & 4 \\
\hline 60. & Iron & 4 \\
\hline 61. & Liquid nitrogen & 4 \\
\hline 62. & Microwave excited electrodeless discharge tube & 4 \\
\hline 63. & Microwave excited emissive detector & 4 \\
\hline 64. & Organic compounds & 4 \\
\hline 65. & Photon & 4 \\
\hline 66. & Tin & 4 \\
\hline 67. & 4(2-pyridylazo) resorcinol & \\
\hline 68. & Absorptiometric & 3 \\
\hline 69. & Acid alizarin black $\mathrm{SN}$ & \\
\hline 70. & Acetylene flames & 3 \\
\hline 71. & Argon & \\
\hline 72. & Atomic absorption & 3 \\
\hline 73. & Bromopyrogallol red & \\
\hline 74. & Catalysis & 3 \\
\hline 75. & Chelating reactions & 3 \\
\hline 76. & Chemical analysis & \\
\hline 77. & EDTA titrations & 3 \\
\hline 78. & Emission spectroscopy & \\
\hline 79. & Flame photometry & 3 \\
\hline 80. & Flame spectroscopy & \\
\hline 81. & Fluoride & 3 \\
\hline 82. & Fluoride ion & \\
\hline 83. & Germanium & 3 \\
\hline 84. & Hair & \\
\hline 85. & Inert gas & 3 \\
\hline 86. & Instrumental methods & \\
\hline 87. & Iron & 3 \\
\hline 88. & Kinetochromic spectrophotometry & 3 \\
\hline 89. & Molecular emission spectroscopy & 3 \\
\hline 90. & Natural waters & 3 \\
\hline 91. & Nitrous oxide acetylene & 3 \\
\hline 92. & Phenylphosphoroxychlorid & 3 \\
\hline 93. & Piezoelectric quartz crystal & 3 \\
\hline 94. & Plants & 3 \\
\hline 95. & Separation methods & 3 \\
\hline 96. & Silicon & 3 \\
\hline 97. & Solvent extraction & 3 \\
\hline 98. & Spectroelectrochemistry & 3 \\
\hline 99. & Tantalum & 3 \\
\hline 100. & Titanium & 3 \\
\hline-101. & Tellurium & 3 \\
\hline
\end{tabular}


Table 4 (Cont.)

\begin{tabular}{|c|c|c|}
\hline $\begin{array}{l}\text { Sr. } \\
\text { No. }\end{array}$ & Keyword & Frequency \\
\hline 102. & Thallium & 3 \\
\hline 103. & Trace analysis & 3 \\
\hline 104. & Tungsten & 3 \\
\hline 105. & Alkali & 2 \\
\hline 106. & Alkaline earth elements & 2 \\
\hline 107. & Aluminium alloys & 2 \\
\hline 108. & Aluminium nutrition & 2 \\
\hline 109. & Atomic emission spectroscopy & 2 \\
\hline 110. & Atomic flame & 2 \\
\hline 111. & Atomic spectrochemical analysis & 2 \\
\hline 112. & Atomic spectrometry & 2 \\
\hline 113. & Atmospheric pressure & 2 \\
\hline 114. & Argon oxygen mixture & 2 \\
\hline 115. & Brass & 2 \\
\hline 116. & Bronze & 2 \\
\hline 117. & Calcein blue & 2 \\
\hline 118. & Carbon monoxide & 2 \\
\hline 119. & Complexometric titration & 2 \\
\hline 120. & Cool flames & 2 \\
\hline 121. & $\begin{array}{l}\text { Cool tlames } \\
\text { Cyanide }\end{array}$ & 2 \\
\hline 122. & $\begin{array}{l}\text { Cyanide } \\
\text { Delves sampling cup }\end{array}$ & 2 \\
\hline 123. & $\begin{array}{l}\text { Delves sampling cup } \\
\text { Dual element electrodeless discharge lamp }\end{array}$ & 2 \\
\hline 124. & $\begin{array}{l}\text { Dual element electrodeless discharge lamp } \\
\text { Electrodeless discharge lamp }\end{array}$ & 2 \\
\hline 125. & $\begin{array}{l}\text { Electrodeless discharge lamp } \\
\text { Electrography }\end{array}$ & 2 \\
\hline 126. & $\begin{array}{l}\text { Electrography } \\
\text { Electronically modulated electrodeless discharge tube }\end{array}$ & 2 \\
\hline 127. & $\begin{array}{l}\text { Electronically modulated electrodeless discharge tube } \\
\text { Emission spectra }\end{array}$ & 2 \\
\hline 128. & $\begin{array}{l}\text { Emission spectra } \\
\text { Ethylenediamine - N,N,N',N'-tetra-n-proionic acid }\end{array}$ & 2 \\
\hline 129. & $\begin{array}{l}\text { Ethylenediamine - N,N,N',N'-tetra-n-proionic acid } \\
\text { Ethylenediamine tetracetic acid }\end{array}$ & 2 \\
\hline 130. & $\begin{array}{l}\text { Ethylenediamine tetracetic acid } \\
\text { Flame emission }\end{array}$ & 2 \\
\hline 131. & $\begin{array}{l}\text { Flame emission } \\
\text { Fluorimetry }\end{array}$ & 2 \\
\hline 132. & $\begin{array}{l}\text { Fluorimetry } \\
\text { Fuel oils }\end{array}$ & 2 \\
\hline 133. & $\begin{array}{l}\text { Fuel oils } \\
\text { Gallium }\end{array}$ & 2 \\
\hline 134. & $\begin{array}{l}\text { Gallium } \\
\text { Graphite furnace }\end{array}$ & 2 \\
\hline 135. & $\begin{array}{l}\text { Graphite furnace } \\
\text { Graphite tube atomizer }\end{array}$ & 2 \\
\hline 136. & $\begin{array}{l}\text { Graphite tube atomizer } \\
\text { Hydrobromic acid }\end{array}$ & 2 \\
\hline 137. & $\begin{array}{l}\text { Hydrobromic acid } \\
\text { High-intensity cathode lamp }\end{array}$ & 2 \\
\hline 138. & $\begin{array}{l}\text { High-intensity cathode lamp } \\
\text { lodine }\end{array}$ & 2 \\
\hline 139. & $\begin{array}{l}\text { Iodine } \\
\text { Indium salts }\end{array}$ & 2 \\
\hline 140. & $\begin{array}{l}\text { Indium salts } \\
\text { Low pressure flames }\end{array}$ & 2 \\
\hline 141. & $\begin{array}{l}\text { Low pressure flames } \\
\text { Lubricating oils }\end{array}$ & 2 \\
\hline 142. & $\begin{array}{l}\text { Lubricating oils } \\
\text { Metal ions }\end{array}$ & 2 \\
\hline 143. & & 2 \\
\hline 144. & Metallochromic indicator & 2 \\
\hline 145. & Molecular emission spectrometry & 2 \\
\hline 145. & Nitrogen sheathed nitrous oxide acetylene flame & \\
\hline
\end{tabular}


Table 4 (Cont.)

\begin{tabular}{rlr}
\hline Sr. & \multicolumn{1}{c}{ Keyword } & Frequency \\
No. & & 2 \\
\hline 146. & Nitrous oxide & 2 \\
147. & Non aqueous media & 2 \\
148. & Ortho-ortho-dihydroxyazo dyes & 2 \\
149. & Orthophosphate & 2 \\
150. & Palladium & 2 \\
151. & Platinum electrode & 2 \\
152. & Qualitative inorganic analysis & 2 \\
153. & Salicylidene-O-aminophenol & 2 \\
154. & Scandium & 2 \\
155. & Spectra-line sources & 2 \\
156. & Spectrofluorescence & 2 \\
157. & Sulphate & 2 \\
158. & Sulphide ion & 2 \\
159. & Thorium & 2 \\
160. & Trace metals & 2 \\
161. & Ultramicro atomic absorption spectrometry & \\
162. & Zirconium methylthymol & \\
\hline
\end{tabular}

The duty of scientists and technologists is not only to conduct their own work at the highest possible level of quality but also to make the societies in which they live more aware of the powers and climate of science. Since by definition, scientists and technologists are the first to known of scientific advances they can be described as the cartographers of the future. They must work to ensure that the public, and especially the public's leaders, understand the maps that they draw. The options which are possible and sustainable, so that wise democratic decision can be taken. ${ }^{13}$

Max Perutz, one of Britain's most distinguished scientists in his book Is Science Necessary: Essays on Science and Scientists states: "In science, as in other fields of endeavour, one finds saints and charlatans, warriors and monks, geniuses and cranks, tyrants and slaves, benefactors and misers, but there is one quality that the best of them have in common, one that they share with great writers, musicians, and artists: creativity". On the other hand there must be caution so as not to uphold orthodox scientists who are as adamant as religious fundamentalists on resisting new ideas destructive to some of their own pet theories and systems of thought. However, it takes belief in ones own ability and ideas, and courage to be a potential rising star and it takes a special kind of person like T.S. West to push out the frontiers of knowledge beyond the current state of the art. 
So far a very few scientometric studies have been carried out on individual scientists. ${ }^{14-36}$ This is an interesting interdisciplinary domain which reveals characteristics of the School of Scientific Excellence, formed around a creative scientist, that results in synergistic effect of progress in Science and Technology. Hence needs further research so as to get incite into the system of harnessing effectively the potentials in human resources. There is a need to assess the performance on a continuous basis. There are individual scientists who have been dòing good work. If we can project image of such scientists, it may be possible to attract talents of younger generation. It is the human intelligence and imagination that is going to build 21st century.

\section{Conclusion}

Publication productivity analysis of the successful scientist, T. S. West, carried out here has thrown light on his pivotal contributions to science and technology. He can be considered as a "role model" for younger researchers to follow. Knowledge is valuable for its own sake and research has cultural value. Desire of being creative is built in our genes. Who knows this effort may switch on genes for creativity in some of those who happen to read this article. Narrating success stories have an encouraging effect. New ways to motivate scientists seems as important to contest outcome as new sources of funds. Science policy makers interested to know about functioning of active research teams as centres of excellence and factors responsible for optimising, maximising and enhancing outputs may appreciate quantitative and qualitative aspects of scientometrics.

It is further suggested that citation analysis of publications of $T$. S. West should be undertaken to know the impact of his research.

\section{References}

1. H. A. LAITINEN, History of analytical chemistry in the USA, Talanta, 36 (1989) 1-9.

2. R. W. MURRAY, Teaching of analytical chemistry in the US, Talanta, 36 (1989) 11-17.

3. C. L. BORGMAN, R. E. RICE, The convergence of information science and communication: A bibliometric analysis, Journal of American Society of Information Science, 43 (1992) 397-411.

4. N. Pravile, C. Oluic-Vukovic, Dual approach to multiple authorship in the study of collaboration/scientific output relationship, Scientometrics, 10 (1986) 259-280.

5 K. Subramanyam, Bibliometric studies of research collaboration: a review, Joumal of Information Science, 6 (1983) 33-38.

6. P. VINKIER, Bibliometric analysis of publication activity of a scientific research institute, In: Informetrics 89/90, L. EGGHE, R. RoussEAU (Eds), Elsevier science publishers, B. V., 1990, pp. $309-334$. 
7. H. WhITE, K. W. MCCAIN, Bibliometrics, Annual Review of Information Science and Technology (ARIST), M. E. WILliams (Ed.), Elsevier science publishers, B. V., 24 (1989) 119-186.

8. L. EGGHE, R. Rousseau, Reflections on a deflection: a note on different causes of the Groos droop, Scientometrics, 14 (1988) 493-511. Bio

9. S. K. SEN, S. K GAN, Bibliometrics: concept and application in the study of productivity, International Forum for Information and Documentation, 15 (1990) 13-21.

10. M. Kunz, Lotka and Zipf: Papers dragon with fuzzy tails, Scientometrics, 13 (1988) 289-297.

11. L. EGGHE, On the $80 / 20$ rule, Scientometrics, 10 (1986) 55-68.

12. L. H. MAIER, Science and the humanities - a plea for interdisciplinary communication, Interdisciplinary Science Reviews, 17 (1992) $171-177$.

13. L. DaInTON, Knowledge is our destiny, Interdisciplinary Science Reviews, 17 (1992) 116-119.

14. P. S. Nagpaul, S. P. GuPTA, Effect of professional competence, managerial role and status of group leaders to R \& D performance, Scientometrics, 17 (1989) 301-331.

15. V. L. KALYANE, S. V. KALYANE, Scientometric portrait of M. S. Swaminathan, Library Science with a Slant to Documentation and Information Studies, 31 (1994) 31-46.

16. B. S. Kademani, V. L. Kalyane, M. R. Balakrishnan, Scientometric portrait of P K. Iyengar, Library Science with a Slant to Documentation and Information Studies, (1994) /55-176.

17. V. L. Kalyane, M. B. Hanil, S. V. Kalyane, Scientific school of a Botanist, International Dr. P. N. Kaula Felicitation Festschrift, Lucknow, (1994) (in press).

18. V. L. KALYANE, R. S. DevaraI, Informetrics on C. S. Venkata Ram, In: New Horizons in Library and Information Science, Dr. Velaga Venkatappaiah Festschrift edited by C. P. VASHISHTH, L. S. RAMAIAH, N. V. Jaggarao, T. V. Prafulla Chandra, T. R. Publications, Madras, (1994) 475-478.

19. V. L. Kalyane, R. K. Samanta, Informetrics on K. Ramiah, Prof. G. V. S. L. Narasimha Raju Festschrift, Vikas Publications, New Delhi,(199,5) $465-478.2$

20. V. L. Kalyane, S. V. Kalyane, Scientometric portrait of Vinodini Reddy, Joumal of Information Sciences, (1993) 25-47.

21. I. RuFf, Citation analysis of a scientific career: A case study, Social Studies of Science, 9 (1979) $81-90$.

22. D. K. GuptA, Citation analysis: A case study of a most cited author and most cited article on Seafloor spreading, LASLIC Bulletin, 28 (1983) 1-12.

23. D. K Gupta, S. GuptA, A citography on LePichon's article on Sea-floor spreading and continent drift: Application of Bradford's law, LASLIC Bulletin, 28 (1983) 49-58.

24. S. C. Sinha, I. M. S. Bhatnagar, The information profile of a plant pathologist: A bibliometric study, Annals of Library Science and Documentation, 21 (1980) 106-113.

25. S. C. Sinha, M. F. Ulla, Citation profile of Dr. V. S. Ramachandran: A bibliometric analysis of his highly cited articles and books in the area of cement and concrete chemistry, Annals of Library Science and Documentation, 40 (1993) 21-31.

26. Z. M. Mulchenko, Yu. V. GranovsKY, A. B. Strakhov, On scientometrical characteristics on information activities of leading scientists, Scientometrics, 26 (1979) 307-325.

27. Y. GUAY, Emergence of basic research on the periphery. Organic Chemistry in India, 1907-1926, Scientometrics, 10 (1986) 77-94.

28. N. SAROJAM, Bibliometric analysis of Indian forest entomology literature, Library Science with a Slant to Documentation and Information Studies, 26 (1989) 228-244.

29. S. S. MunNoli, V. L. Kalyane, Scientometric portrait of R. G. Rastogi, ILA Bulletin, (1995) (in press).

30. B. S. Kademani, V. L. Kalyane, A. B. Kademani, Scientometric portrait of Nobel Laureate Dr. C. V. Raman, Indian Joumal of Information, Library, and Society, 7 (1994) 215 - 249.

31. D. K. GuPTA, Plate tectonics: a case study of transmission of ideas, Annals of Library Science and Documentation, 25 (1978) 86-92.

32. T. CAWKELL, E. GARFIELD, Assessing Einstein's impact on today's science by citation analysis, In: Einstein: the First Hundred Years, M. Goldsmith, A. MACKAY, J. WoudHuYsEN (Eds), Pergamon press, Oxford, 1980, pp. 31-40.

33. D. K. GUPTA, Chandrasekhar: winner of the 1983 Nobel prize for physics: A citation analysis study of his works, Annals of Library Science and Documentation, 30 (1983) 177-184.

34. H. KraGH, Dirac bibliometrics. In: Dirac: A Scientific Biography, Cambridge University press, Cambridge, 1990, pp. 293-301. 
35. R. TOdorov, M. WINTERHAGER, An overview of Mike Moravcsik's publication activity in physics, Scientometrics, 20 (1991) 163-172.

36. K. C. GARG, M. M. S. KARK, Bibliometrics of research communications of INSA fellows, Joumal of Scientific and Industrial Research, 51 (1992) 929-935.

37. A. B. KaDEMANI, B. S. KADEMANI, V. L. KALYANE, Scientometric portrait of $\mathrm{R}$. S. Krishnan, Indian Jaurnal of Information, Library and Societ, \& (1995) 